\title{
$\nabla$ \\ Breast Carcinoma in Axillary Tail of Spence: A Rare Case Report
}

\section{IJCRR}

Section: Healthcare

Sci. Journal Impact

Factor: 6.1 (2018)

ICV: 90.90 (2018)

Scopus'

\section{Rajesh Domkunti ${ }^{1}$, Yashwant R. Lamture ${ }^{2}$, Avinash Rinait ${ }^{3}$, Dilip Gode ${ }^{4}$}

Jawaharlal Nehru Medical College, Datta Meghe Institute of Medical Sciences, Wardha-442001; 2 Professor and HOD Dept. of Surgery, Datta Meghe Medical College Nagpur, Shalinitai Meghe Hospital and Research Centre, Nagpur-441110; ${ }^{3}$ Assistant Professor Dept. of Surgery, Datta Meghe Medical College Nagpur, Shalinitai Meghe Hospital and Research Centre, Nagpur-441110; ${ }^{2}$ Dean \& Professor Dept. of Surgery, Datta Meghe Medical College Nagpur, Shalinitai Meghe Hospital and Research Centre, Nagpur-441110

\section{ABSTRACT}

Breast carcinoma is known to be one of the commonest malignant tumours among which Axillary breast cancer is a special individual that accounts for $0.1 \%$ to $2 \%$ of all cases of breast cancer. This is usually composed of poorly differentiated IDC with squamous or mesenchymal carcinoma areas sometimes. Axillary malignancy is difficult as there are many differential diagnoses that may require careful evaluation. This is a case report on Breast carcinoma of tail of Spence in axilla with skin involvement near nipple-areola complex whose histopathology was suggestive of infiltrating ductal carcinoma of axillary tail of Spence with mild dysplastic changes over right nipple-areola complex and high lymph node positivity (96.7\%). Standard investigations like Ultrasonography of B/L Breast with Axilla and Tru-cut biopsy were done to reach the diagnosis of Intraductal Carcinoma (IDC) of the axillary tail.

Key Words: Tru-cut biopsy, Ultrasonography, High lymph node positivity, Intraductal carcinoma of breast

\section{INTRODUCTION}

Breast carcinoma is known to be one of the commonest malignant tumors. The researchers have confirmed various characteristics of independent factors that affect the prognosis, such as histologic type, age, ER, PR, grade, etc. As per the studies, the commonest site for breast cancer is Upper Outer Quadrant (UOQ) which accounts for $39-57 \%$ of total cases and the rarest site is Lower Inner Quadrant (LIQ) which accounts for $7-10 \%$ of total cases. ${ }^{5}$ Axillary carcinoma of breast is a rare, accounting for $0.1 \%$ to $2 \%$ of all cases of breast carcinoma. Typically it consists, poorly differentiated IDC with intermittent areas of squamous or mesenchymal carcinoma. ${ }^{3}$ The axillary tail of Spence (ATS) is of variable size and it can be sighted as a narrow extension in the upper outer quadrant of the breast (UOB) gland. The mammary gland of the ATS starts from the UOB extending to enter the axilla through a defect in the clavipectoral fascia. Due to the special anatomical position of ATS, the lymph nodes (LNs) are similar to each other and can also be located in the axillary tail region is a unique and poorly studied type of cancer of the breast. ${ }^{5}$ The axillary tail region contains breast tissue and lymph nodes ${ }^{2}$, so the reported differential diagnoses of CATS are occult breast carcinoma, accessory breast cancer, axillary tail breast cancer, and lymphoma or non-breast metastatic lymphadenopathy. ${ }^{3}$ Surgical management of the disease (breast conservation therapy or modified radical mastectomy) was left to the surgeon's judgment because the tail of Spence is an essential extension of the breast, and surgery the mainstay of treatment. It is necessary for all patients to have non-disseminated disease to undergo adjuvant chemotherapy with doxorubicin-containing regimens ${ }^{2}$. CATS has a prelidiction towards advanced stage and hormone receptor-negative neoplasms. The recurrence of cancer in the case of ATS cancer after prophylactic mastectomy is high. It is challenging to establish the prognosis of CATS, due to none or limited follow-up of cases, as well as the small sample sizes due to fewer cases of CATS, and also there is no specific guideline for it. The effect of the location of tumor in the axillary tail, on survival rates of patients, has not been thoroughly explored yet. (5) In this case report, we share our experience of clinical assessment, diagnosis, management, histopathology assessment of a breast carcinoma in the axillary tail of spence which is a very rare and unique entity.

\section{Corresponding Author:}

Dr. Yashwant R. Lamture, Professor Department of Surgery, Datta Meghe medical college Nagpur, Shalinitai Meghe Hospital and Research Centre, Nagpur-441110

ISSN: 2231-2196 (Print) ISSN: 0975-5241 (Online)

Received: 28.05 .2020

Revised: 21.06 .2020

Accepted: 03.07.2020

Published: 21.07 .2020 


\section{CASE REPORT}

A 50 year old female came with complaints of mass in the right the axillary region for 3 months. She also presented with a wound over the right nipple areolar complex. The patient is a known case of Type-II Diabetes Mellitus and Systemic Hypertension since 6 years and was on regular medication (Tab. Metformin 500mg twice daily and Amlodepine-Atenolol $\{5 / 50 \mathrm{mg}\}$ once daily). Patient procured menarche at 12 years of age and had regular menstrual cycles and gave birth to two children (first child was born at the age of 24 years) and attained Menopause at the age of 45 years. She has breastfed all the kids for 9 months. Patient gives no history of any surgery, hormonal replacement therapy in the past. Patient gives no history of similar complaints in the family members. General examination was unremarkable. Local examination: left breast and axilla was unremarkable. Right breast showed ulcerated nipple areolar region measuring $3 \times 3.5 \mathrm{~cm}$ with pale pink granulation tissue and blackened surrounding skin. Mass of $5 \times 4.5 \mathrm{~cm}$ was palpable in the right axillary region which was firm, fixed, non-tender with no skin changes (See Fig. 1A and 1B).

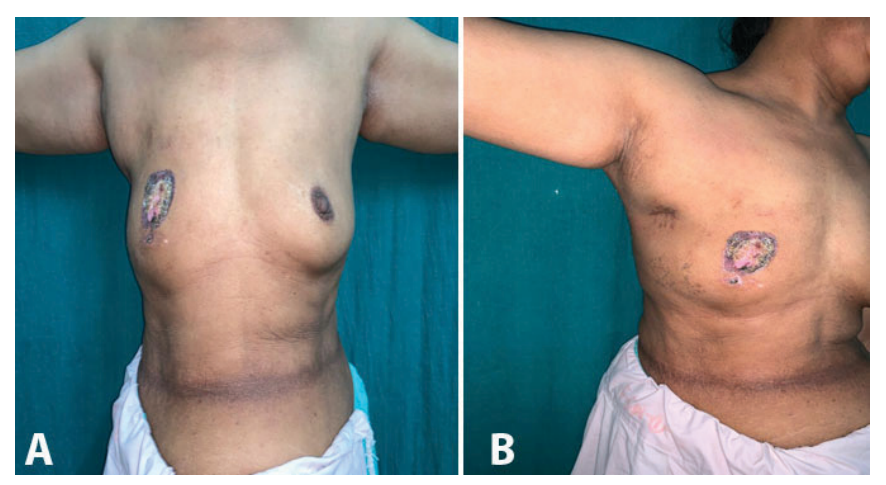

Figure 1(A \& B): Clinical photograph showing right axillary lump and ulcerated wound over right Nipple Areolar Complex.

Routine blood investigations were normal (Complete blood count, Liver function test, Kidney function test, Random blood sugar, coagulation profile, urine routine, and microscopy). On Day 1 of admission Ultrasonography of B/L Breast and Axilla was done which showed ill-defined hypoechoic lesion in the right nipple-areola complex region measuring $0.5 \mathrm{~cm}$ along with the heterogenous hypoechoic vascular lesion in the right axilla measuring $4 \times 3 \mathrm{~cm}$ with multiple lymphadenopathy suggestive of neoplastic etiology and Metastatic workup (Ultrasonography of abdomen and pelvis, X-ray of chest and thoraco-lumbar spine) was done which was unremarkable. On Day 2 of admission, Tru-cut Biopsy was taken from the ulcer and axillar mass of right breast and reporting was done on Day 6 of admission which was suggestive of Carcinoma of Axillary tail of spence with skin involvement and nodal metastasis. Oncologist opinion was taken on the same day and was advised surgery followed by 4 cycles of adjuvant chemotherapy (AC regimen followed by Paclitaxel). On day 7 of admission Patient underwent right modified radical mastectomy (See Fig 2) and the specimen was sent for histopathological examination (See Fig 3).

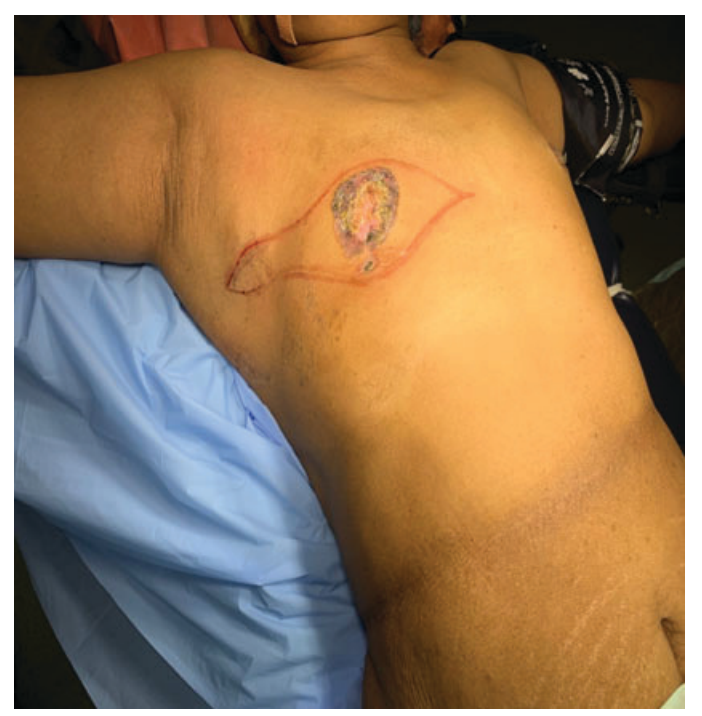

Figure 2: Clinical photograph showing the markings done for surgical resection of right axillary lump along with ulcerated wound.

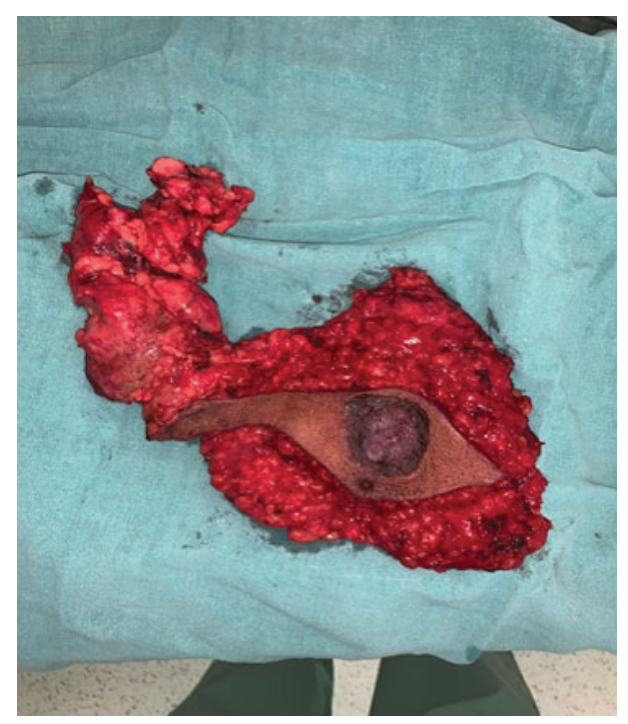

Figure 3: photograph showing the excised specimen sent for histopathological examination which includes right axillary lump, complete breast tissue along with the part of overlying skin including the ulcerated wound.

On Day 14 of admission histopathology reporting was done which was suggestive of infiltrating ductal carcinoma of size $3 \times 2.5 \mathrm{~cm}$ arising from the axillary tail of spence of the right breast and invading fat tissue with lymphovascular invasion. Section taken from ulcer over nipple-areola complex shows changes suggestive of mild dysplasia. It was accom- 
panied by 31 lymph nodes out of which 30 were positive for malignant cells. The invasive lesion showed the following characteristics: estrogen receptor (ER)-positive, progesterone receptor-positive, and HER2-negative. Post-operatively patient received chemotherapy in the form of $\mathrm{AC}$ regimen (Adriamycin and Cyclophosphamide), followed by Paclitaxel therapy. The patient is currently 16 weeks post-operatively and has no signs of recurrence(See Fig 4).

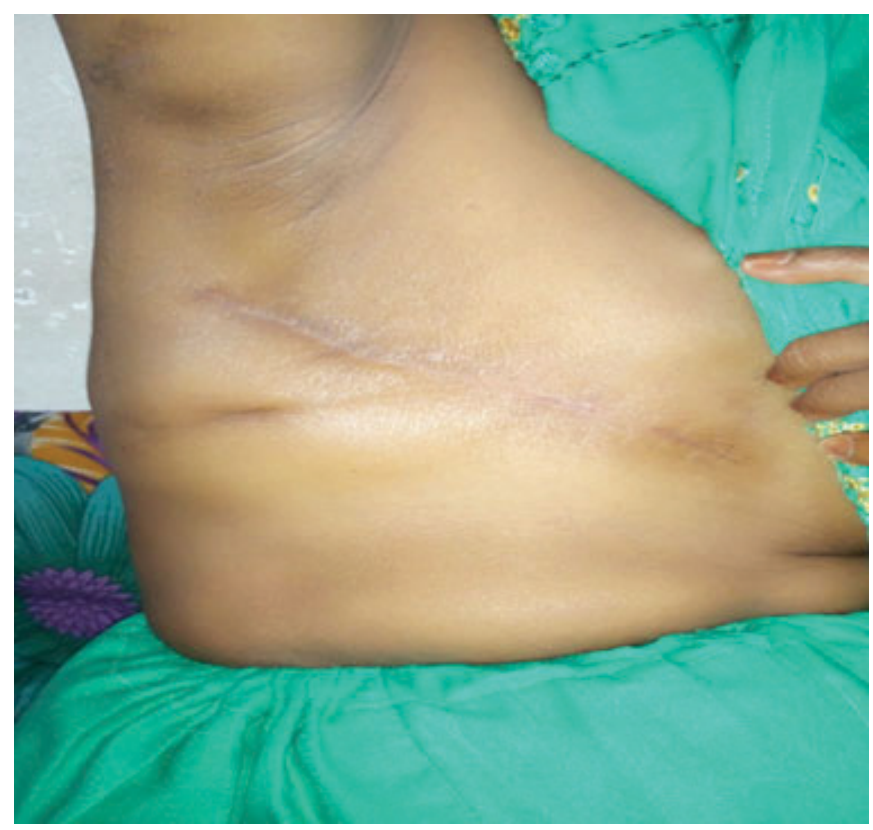

Figure 4: Clinical photograph showing the operated site with healthy scar line after 4 months with no recurrence.

\section{DISCUSSION}

Breast carcinoma in the axillary tail of Spence is very rare. Ampil et al. reported a frequency of this type of breast cancer to be $0.1 \%{ }^{1}$. It possesses atypical clinical, pathological, and prognostic features, promoting the likelihood of nodal metastasis that affects both disease progression and survival ${ }^{3}$. This case report is unique due to two of its characteristicsOne is the axillary tail of spence being the site of origin of tumour and the other is the involvement of nipple-areola complex of the same breast having mild dysplastic changes leading to the formation of the wound.

Previous research reveals that most patients having CATS are in the old $(>45 \text { years })^{5}$. In our case the age of the patient was 50 years. As per studies, patients usually discover the mass on self-examination and seek medical advice ${ }^{3}$. In our case patient presented with mass in the right axilla and wound causing distortion of nipple-areola complex of the right breast.

In many studies, the standard modalities for investigations are ultrasound or mammography in the case of $\mathrm{CATS}^{3}$. In our case patient underwent Ultrasonography of $\mathrm{B} / \mathrm{L}$ Breast with axilla which showed ill-defined hypoechoic lesion in the right nipple-areola complex region measuring $0.5 \mathrm{~cm}$ along with the heterogenous hypoechoic vascular lesion in the right axilla measuring $4 \times 3 \mathrm{~cm}$ with multiple lymphadenopathy suggestive of neoplastic etiology.

As per previous research Ultrasound-guided fine-needle aspiration biopsy is the first step to differentiate benign from malignant lesions ${ }^{4}$. In our case, Tru-cut Biopsy was taken from the ulcer and axillary mass of right breast which was suggestive of Carcinoma of Axillary tail of spence (CATS) with skin involvement as well as nodal metastasis.

As per standard guidelines, surgery remains the main CATS Treatment. Operational control is subject to the Surgeon's discretion $^{5}$, which consists of wide local excision, regional lymph node dissection, and radical mastectomy of the ipsilateral breast if it is involved ${ }^{4}$. In our case patient underwent Right Modified Radical Mastectomy (MRM) as there was the involvement of skin around Nipple-Areola complex.

As per the study by Mohammas A. Nouh et al. the highest node positivity was observed in secretory, adenosquamous, ductal and lobular carcinomas in a descending order where it varied between $100 \%$ and $72.3 \%{ }^{6}$. In our case, histologic type was ductal carcinoma and lymph node positivity was $96.7 \%$ (31 lymph nodes were identified out of which 30 lymph nodes were positive).

\section{CONCLUSION}

In conclusion carcinoma in axillary tail of spence is a unique and challenging entity that needs special attention in clinical, diagnosing, managing, and pathological practice. We have reported a very unique case of Breast carcinoma in the axillary tail of Spence along with the involvement of nippleareola complex having mild dysplastic changes with histopathology report revealing infiltrating ductal carcinoma type with high nodal positivity.

\section{Acknowledgement: None}

Conflict of interest: None

Financial support: None

\section{REFERENCES}

1. Okubo M, Tada K, Niwa T, Nishioka K, Tsuji E, Ogawa T, et al., A Case of breast cancer in the axillary tail of spence - enhances magnetic resonance imaging and positron emission tomography for diagnostic differentiation and preoperative treatment decision, World J Surg Oncol, 2013 ; 11(217) : 1-5.

2. Ampil F, Caldito G, Henderson B, Li B, Kim RH, Burton G, et al., Carcinoma of the axillary tail of spence: a case series, Anticancer Res, 2012 ; 32(9) : 4057-4059. 
3. Hadi MA, Shammari E, AI Sinan S, Shahbahai R, AI Khwaja K, AI Gowejez R, et al., Axillary tail breast cancer: report of two cases and review of literature, Clin Surg, 2019 ; 4(2375) : 1-2.

4. Virgili A, Trincone S, Durante E, Corazza M, Breast cancer of the axillary extension, Acta dermato-venereologica, $2005 ; 85(1)$ : 81-82.

5. Gou ZC, Liu XY, Xiao Y, Zhao S, Jiang YZ, Shao ZM, Decreased survival in patients with carcinoma of axillary tail versus upper outer quadrant breast cancers: a SEER population based study, Cancer Manag Res, 2018 ; 10 : 1133-1141.

6. Nouh MA, Ismail H, EI-Din NH, EI-Bolkainy MN, Lymph node metastasis in breast carcinoma : clinicopathological correlations in 3747 patients, J Egypt Natl Canc Inst, 2004; 16(1): 50-56. 